\title{
Skin $\mathrm{T}_{\mathrm{RM}}$ mediates distributed border patrol
}

\author{
Haina Shin ${ }^{1}$, Akiko Iwasaki ${ }^{1}$ \\ IDepartment of Immunobiology, Yale University School of Medicine, New Haven, CT 06520, USA \\ Cell Research (2012) 22:1325-1327. doi:10.1038/cr.2012.75; published online 8 May 2012
}

Tissue-resident memory $T$ cells $\left(T_{R M}\right)$ are a new subset of memory cells that have been associated with enhanced protective immunity for their tissue of residence. A recent study by Jiang et al. sheds light on the migration behavior of $\mathrm{T}_{\mathrm{RM}}$ in both infected and unifected skin, and their ability to provide protection against re-infection even within a previously uninfected skin tissue.

$T$ cell memory is a hallmark of adaptive immunity. Two broad subsets, effector memory $\left(\mathrm{T}_{\mathrm{EM}}\right)$ and central memory $\left(\mathrm{T}_{\mathrm{CM}}\right)$ have been defined largely by their migratory capacities [1]. CD62 $\mathrm{L}^{10}$ $\mathrm{CCR} 7^{\text {lo }} \mathrm{T}_{\mathrm{EM}}$ circulate through peripheral tissues, while $\mathrm{CD} 62 \mathrm{~L}^{\text {hi }} \mathrm{CCR} 7^{\text {hi }} \mathrm{T}_{\mathrm{CM}}$ home to secondary lymphoid tissues $[1,2]$. More recently, another distinct subset, tissue-resident memory $\mathrm{T}$ cells $\left(\mathrm{T}_{\mathrm{RM}}\right)$ have been described. $\mathrm{T}_{\mathrm{RM}}$ have been found in several tissues, including the skin, gut, lung and brain [3-6]. Once established, $\mathrm{T}_{\mathrm{RM}}$ are maintained independently of circulating $\mathrm{T}_{\mathrm{EM}}$ and $\mathrm{T}_{\mathrm{CM}}$ and their presence correlates with superior protection against local viral challenge $[3,5,7]$. However, the exact role of $T_{R M}$ vs. recruited circulating memory $\mathrm{T}$ cells in controlling viral replication is unclear. Furthermore, due to the confined patrolling area of $\mathrm{T}_{\mathrm{RM}}$, the capacity of $T_{R M}$ to mediate protection against infection at distal sites is

\footnotetext{
Correspondence: Akiko Iwasaki

E-mail: akiko.iwasaki@yale.edu
}

unknown.

A recent paper by Xiaodong Jiang et $a l$. [8] investigated these issues in a skin infection model using adoptively transferred TCR transgenic CD8 T cells that recognize the ovalbumin protein (OT-I) and vaccinia virus expressing ovalbumin (VACV). They first characterized the requirements necessary for establishment of $\mathrm{T}_{\mathrm{RM}}$ in the skin, and found that unlike other epithelial tissues such as the female genital tract [9], entry of CD8 T cells into the skin was independent of CD4 T cells and IFN $\gamma$. However, $\mathrm{T}$ cell expression of ligands for $\mathrm{E}-$ and P-selectin, two adhesion molecules that are expressed constitutively and are upregulated on inflamed endothelial cells of skin blood vessels, was necessary for seeding of the skin with OT-I T cells. These data parallel the requirement for circulating $T_{E M}$ CD4 $T$ cell entry into inflamed skin [10]. Furthermore, in agreement with previous studies using a different virus, herpes simplex virus type 1 (HSV-1) [10], the authors found that after VACV infection, while CD8 T cells were retained within the skin, CD4 $\mathrm{T}$ cells were not.

The differential expression of homing molecules such as $\mathrm{CD} 62 \mathrm{~L}$ and CCR 7 on subsets of memory $\mathrm{T}$ cells indicates their preferential migration to lymphoid or non-lymphoid tissues during homeostasis. However, studies using parabiotic mice have shown that memory $\mathrm{T}$ cells that reside in peripheral tissues such as the brain and gut do not participate in homeostatic circulation; rather, they appear to be maintained independently of circulating memory $\mathrm{T}$ cells [7]. By surgically joining VACVimmunized mice with unimmunized partners, the authors found that $\mathrm{T}_{\mathrm{CM}}$ populations in tissues such as the spleen and lymph node equilibrated between the parabionts, while $\mathrm{T}_{\mathrm{RM}}$ populations in the skin did not. This indicated that like the brain and gut, skin $\mathrm{T}_{\mathrm{RM}}$ populations were excluded from the pool of circulating memory $\mathrm{T}$ cells. Additionally, this parabiosis study indicates that like the gut, the skin must be seeded relatively early in the $\mathrm{T}$ cell response in order to establish a $\mathrm{T}_{\mathrm{RM}}$ population [11]. Jiang et al. also note that $\mathrm{T}_{\mathrm{RM}}$ populations, while fully capable of effector function like their $\mathrm{T}_{\mathrm{EM}}$ and $\mathrm{T}_{\mathrm{CM}}$ counterparts, can be further distinguished by their differential expression of surface molecules, such as the lack of markers CD127 and CD122, and high expression of CD69 and CD103.

Other viral infection models such as HSV-1 have suggested that local memory CD8 T cells can be directly activated in peripheral tissues [12] and participate in controlling viral replication [3]. However, it has been difficult to separate the relative contributions of recruited vs. resident memory $\mathrm{T}$ cells in mediating protection. To address this issue, Jiang et al. separated immunized and unimmunized parabiotic pairs once the $\mathrm{T}_{\mathrm{CM}}$ compartment had equilibrated and gave the mice a skin challenge with VACV. The challenged mice were then treated with FTY720, an ${\mathrm{S} 1 \mathrm{P}_{1}}_{1}$ 
modulator that sequesters lymphocytes in secondary lymphoid organs, thus preventing the recruitment of circulating $T$ cells to the site of infection [13]. They discovered that $T_{R M}$ were superior to $T_{C M}$ in controlling a secondary infection, as the immunized parabiont controlled viral replication significantly better than the unimmunized partner. Furthermore, they found no difference in the viral titers of the untreated and FTY720-treated immunized parabionts. This suggests that in the VACV infection model, $\mathrm{T}_{\mathrm{RM}}$ are sufficient to mediate protection against reinfection, even in the absence of any input from circulating $\mathrm{T}$ cells.

When a host is immunized through the skin by methods such as scarification, previous studies have implied that the $T_{R M}$ will remain local and patrol an area of tissue that is directly adjacent to the site of infection. For example, in the HSV-1 infection model, it has been shown that when mice are infected on one flank, that flank is better protected against subsequent challenge than the contralateral, unimmunized flank [3]. Migration of T cells into tissues such as the skin and gut requires the expression of particular homing markers that are most strongly upregulated when $T$ cells are activated via immunization routes that involve the target tissue [14]. Ligands to some of the skin homing markers upregulated by activated $\mathrm{T}$ cells are constitutively expressed in blood vessel endothelium, albeit at a lower level than inflamed tissue. This raises questions as to whether $\mathrm{T}$ cells activated through local skin infection can accumulate in distal unimmunized skin, and whether these $\mathrm{T}$ cells can provide preferential protection compared to $\mathrm{T}$ cells activated through other immunization routes. To address these questions, Jiang et al. immunized mice on one ear using VACV and then examined the accumulation of $\mathrm{T}$ cells in the other ear. They found that activated OT-I cells were capable of migrating to the uninfected ear, and that the number of cells found in the

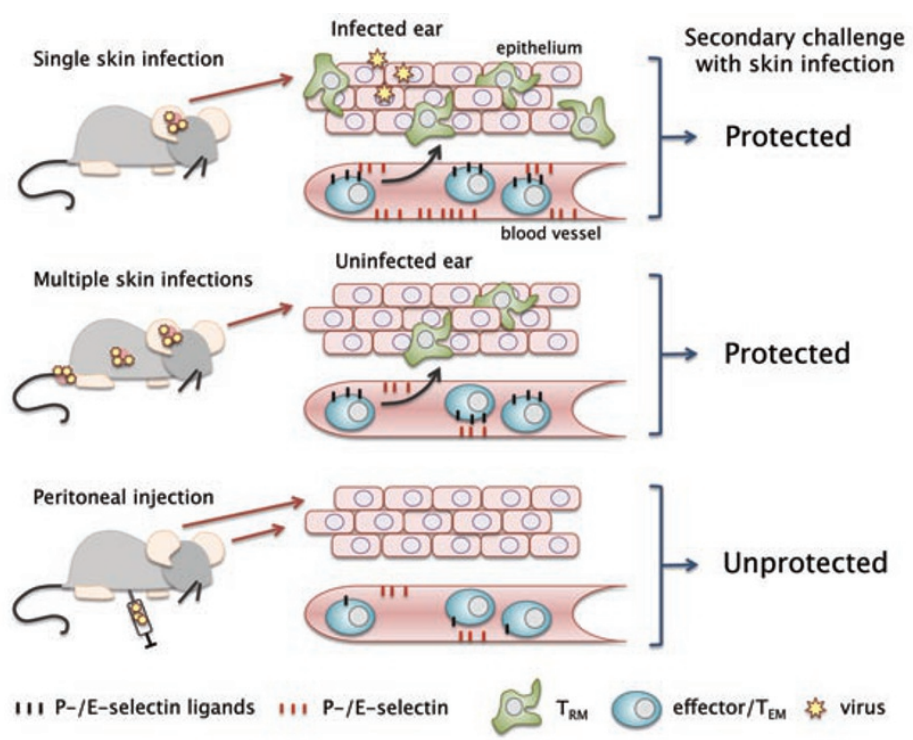

Figure 1 Skin epidermal infection by virus leads to $T_{R M}$ residency and local or global antiviral protection. When the host is infected via skin scarification, the resulting inflammation increases expression of chemoattractants and adhesion molecules such as P- and E-selectin on the endothelium of the blood vessels. $\mathrm{T}$ cells activated through this route upregulate skin homing markers such as $\mathrm{P}$ - and E-selectin ligands. Collectively, these events lead to the recruitment of circulating, antigen-specific effector/effector memory $\left(T_{E M}\right)$ CD8 $T$ cells into the infected tissue. The recruited CD8 $T$ cells establish a pool of resident memory $T$ cells $\left(T_{R M}\right)$ that can provide protection against future skin infections. Jiang et al. show that upon multiple skin immunizations, circulating effector/ $T_{E M}$ CD8 T cells also accumulate at uninfected sites. These CD8 $T$ cells can then form a $\mathrm{T}_{\mathrm{RM}}$ population that is immunologically protective. However, the establishment of $T_{R M}$ in uninfected skin is dependent on the route of immunization, as skinindependent methods such as intraperitoneal injection did not lead to protection against a secondary skin challenge.

uninfected ear could be boosted by immunizing mice multiple times through various skin routes. Furthermore, when mice were challenged in the previously uninfected ear, viral titers were significantly lower in mice immunized via skin scarification compared to mice immunized intraperitoneally (Figure 1). Together with the $\mathrm{T}$ cell number data, the authors suggest that $\mathrm{T}_{\mathrm{RM}}$ in the skin can be established at sites distal to the initial infection, and that these $\mathrm{T}_{\mathrm{RM}}$ may play a role in providing widespread protection against reinfection.

In summary, the study by Jiang and colleagues show that $\mathrm{T}_{\mathrm{RM}}$ established in the skin are maintained independently of circulating $\mathrm{T}_{\mathrm{CM}}$. Furthermore, they demonstrate that $\mathrm{T}_{\mathrm{RM}}$ alone are sufficient to protect against pathogens such as vaccinia virus, although the role that different subsets of memory T cells play in mediating immunity will likely vary depending on the infectious agent [10, 15]. Importantly, Jiang et al. show that through prime-boosting, a substantial $\mathrm{T}_{\mathrm{RM}}$ population can be established at distal skin sites absent of infection or inflammation. This suggests that the frontline defense that $T_{R M}$ can provide in barrier tissues does not have to be limited by the location of immunization (Figure 1). In future studies, it will be important to continue to gain an understanding of $\mathrm{T}_{\mathrm{RM}}$, including the factors that lead to their establishment and maintenance. 
Despite the variety of tissues in which $T_{R M}$ have been described, $T_{R M}$ appear to share certain phenotypic and functional characteristics, such as the expression of surface markers CD103 and CD69, as well as the ability to produce effector molecules such as IFN $\gamma$ and granzyme B $[5,6,8,16]$. Thus, it will be interesting to determine whether there is a common pathway by which $\mathrm{T}_{\mathrm{RM}}$ differentiate, or whether each tissue has its own individual requirements. Beyond establishment, the mechanism that mediates the long-term survival of $\mathrm{T}_{\mathrm{RM}}$ is unknown. Previous studies have shown that $T_{R M}$ from tissues such as the brain do not survive well when taken out of their tissue of residence [6]. This suggests that the signals that sustain $T_{R M}$ may be locally provided and may even be tissue specific, different from the cytokines that maintain systemic $\mathrm{T}_{\mathrm{CM}}$ and $\mathrm{T}_{\mathrm{EM}}$. Understanding how $\mathrm{T}_{\mathrm{RM}}$ are established and the roles that they play in protecting against invading pathogens will be critical in designing vaccines and immunotherapies that target specific tissues.

\section{References}

1 Sallusto F, Lenig D, Forster R, Lipp M, Lanzavecchia A. Two subsets of memory $\mathrm{T}$ lymphocytes with distinct homing potentials and effector functions. Nature 1999; 401:708-712.
2 Masopust D, Vezys V, Marzo AL, Lefrancois L. Preferential localization of effector memory cells in nonlymphoid tissue. Science 2001; 291:2413-2417.

3 Gebhardt T, Wakim LM, Eidsmo L, Reading PC, Heath WR, Carbone FR. Memory T cells in nonlymphoid tissue that provide enhanced local immunity during infection with herpes simplex virus. Nat Immunol 2009; 10:524-530.

4 Kim SK, Schluns KS, Lefrançois L. Induction and visualization of mucosal memory CD8 T cells following systemic virus infection. J Immunol 1999; 163:4125-4132.

5 Teijaro JR, Turner D, Pham Q, Wherry EJ, Lefrançois L, Farber DL. Cutting edge: Tissue-retentive lung memory CD4 T cells mediate optimal protection to respiratory virus infection. J Immunol 2011; 187:5510-5514.

6 Wakim LM, Woodward-Davis A, Bevan MJ. Memory $\mathrm{T}$ cells persisting within the brain after local infection show functional adaptations to their tissue of residence. Proc Natl Acad Sci USA 2010; 107:17872-17879.

7 Klonowski KD, Williams KJ, Marzo AL, Blair DA, Lingenheld EG, Lefrançois L. Dynamics of blood-borne CD8 memory $\mathrm{T}$ cell migration in vivo. Immunity 2004; 20:551-562.

8 Jiang X, Clark RA, Liu L, Wagers AJ, Fuhlbrigge RC, Kupper TS. Skin infection generates non-migratory memory CD8+ $\mathrm{T}(\mathrm{RM})$ cells providing global skin immunity. Nature 2012; 483:227231.

9 Nakanishi Y, Lu B, Gerard C, Iwasaki
A. CD8+ T lymphocyte mobilization to virus-infected tissue requires CD4+ Tcell help. Nature 2009; 462:510-513.

10 Gebhardt T, Whitney PG, Zaid A, et al. Different patterns of peripheral migration by memory CD4+ and CD8+ T cells. Nature 2011; 477:216-219.

11 Masopust D, Choo D, Vezys V, et al. Dynamic $\mathrm{T}$ cell migration program provides resident memory within intestinal epithelium. J Exp Med 2010; 207:553564.

12 Wakim LM, Waithman J, van Rooijen N, Heath WR, Carbone FR. Dendritic cell-induced memory $\mathrm{T}$ cell activation in nonlymphoid tissues. Science 2008; 319:198-202.

13 Matloubian M, Lo CG, Cinamon G, et al. Lymphocyte egress from thymus and peripheral lymphoid organs is dependent on $\mathrm{S} 1 \mathrm{P}$ receptor 1. Nature 2004; 427:355-360.

14 Woodland DL, Kohlmeier JE. Migration, maintenance and recall of memory $\mathrm{T}$ cells in peripheral tissues. Nat Rev Immunol 2009; 9:153-161.

15 Bachmann MF, Wolint P, Schwarz K, Jager $\mathrm{P}$, Oxenius A. Functional properties and lineage relationship of CD8+ T cell subsets identified by expression of IL-7 receptor alpha and CD62L. J Immunol 2005; 175:4686-4696.

16 Masopust D, Vezys V, Wherry EJ, Barber DL, Ahmed R. Cutting edge: gut microenvironment promotes differentiation of a unique memory CD8 T cell population. J Immunol 2006; 176:2079-2083. 